\title{
BADLY ORDERED CYCLES OF CIRCLE MAPS
}

\author{
Lluís AlsedÀ, Jaume Llibre And Michą Misiurewicz
}

\begin{abstract}
A cycle of a circle map of degree one is badly ordered if it cannot be divided into blocks of consecutive points, such that the blocks are permuted by the map like points of a cycle of a rational rotation. We find the smallest possible rotation intervals that a map with a badly ordered cycle of a given rotation number and period can have. Moreover, we show that if one of those intervals is contained in the interior of the rotation interval of a map then the map has a corresponding badly ordered cycle.
\end{abstract}

\section{Introduction.}

It is always very useful to be able to derive many consequences from a few bits of information. This situation arises in one dimensional dynamics, when knowing ordering of points along a cycle (a periodic orbit) we can often say a lot about a system. Here we want to study what happens if our system consists of iterations of a continuous degree one circle map and the information we have is that there is a badly ordered cycle of a given period and rotation number. The information we want to get is how large the rotation interval of the map has to be. This is the most important information. Once we know the rotation interval, we can estimate the set of periods and topological entropy (see e.g. [1]).

To state our assumptions and results in a more rigorous way, we introduce some notation in spirit of $[1]$. We will consider the circle $\mathbb{S}^{1}$ as $\{z \in \mathbb{C}:|z|=$ $1\}$ where $\mathbb{C}$ denotes the complex plane. Then we will denote by $e: \mathbb{R} \longrightarrow \mathbb{S}^{1}$ the natural projection $e(X)=\exp (2 \pi i X)$, where $\mathbb{R}$ denotes the real line. A continuous map $F: \mathbb{R} \longrightarrow \mathbb{R}$ is called a lifting of a continuous map $f: \mathbb{S}^{1} \longrightarrow \mathbb{S}^{1}$ if $e \circ F=f \circ e$. It can be seen that if $f$ is a continuous map of the circle into itself of degree one and $F$ is a lifting of $f$ then $F(X+1)=F(X)+1$ for all $X \in \mathbb{R}$ and that $F(X+k)=F(X)+k$ for all $X \in \mathbb{R}$ and $k \in \mathbb{Z}$, where $\mathbb{Z}$ denotes the set of all integers. In the sequel, the class of all liftings of continuous maps of the circle into itself of degree 1 will be denoted by $\mathcal{L}$.

We shall say that a point $X \in \mathbb{R}$ is periodic (mod. 1) of period $s$ for a $\operatorname{map} F \in \mathcal{L}$ if $F^{s}(X)-X \in \mathbb{Z}$ but $F^{i}(X)-X \notin \mathbb{Z}$ for $i=1, \ldots, s-1$. We note that if $F$ is a lifting of $f$ then $X$ is periodic (mod. 1 ) for $F$ if and 
only if $e(X)$ is periodic for $f$ and their periods are equal. Let $X \in \mathbb{R}$ and let $F \in \mathcal{L}$. The orbit (mod. 1 ) of $X$ is defined to be the set $\left\{F^{n}(X)+m\right.$ : for $n \geq 0$ and $m \in \mathbb{Z}\}$. The orbit (mod. 1) $P$ of a periodic (mod. 1$)$ point of period $s$ will be called a cycle (mod. 1$)$ of $F$ of period $s$.

Next we shall introduce the notion of a badly ordered cycle which will play a key role throughout the paper. To this end, we will introduce the notions of a twist cycle and of a block structure.

Let $P$ be a cycle (mod. 1 ) of a map $F \in \mathcal{L}$. We say that $P$ is twist if $\left.F\right|_{P}$ is increasing.

Let $P=\left\{X_{i}\right\}_{i \in \mathbb{Z}}$ be a cycle $(\bmod .1)$ of a map $F \in \mathcal{L}$ of period $p$ and let $Q=\left\{Y_{i}\right\}_{i \in \mathbb{Z}}$ be a cycle (mod. 1) of a map $G \in \mathcal{L}$ of period $q$. Assume that $X_{i}<X_{j}$ and $Y_{i}<Y_{j}$ if and only if $i<j$. We say that $P$ has a block structure over $Q$ if $p=n q$ for some $n \in \mathbb{N}$ and there is $k \in \mathbb{Z}$ such that $P=\bigcup_{i \in \mathbb{Z}} P_{i}$, where $P_{i}=\left\{X_{k+i n}, X_{k+i n+1}, \ldots, X_{k+(i+1) n-1}\right\}$ for $i \in \mathbb{Z}$ and $F\left(P_{i}\right)=P_{j}$ if and only if $G\left(Y_{i}\right)=Y_{j}$.

Lastly, a cycle (mod. 1) will be called badly ordered if it has no block structure over a twist cycle. We note that the notions of a twist cycle, block structure and badly ordered cycle are independent of the lifting.

Next we shall introduce the notion of rotation interval of a map from $\mathcal{L}$.

Let $F \in \mathcal{L}$. For $X \in \mathbb{R}$ we define its rotation number as

$$
\limsup _{n \rightarrow \infty} \frac{F^{n}(X)-X}{n},
$$

and we denote it by $\rho(X)$ or $\rho_{F}(X)$ (see [8]). We note that if $X$ is a periodic (mod. 1) point of $F$ with period $s$ such that $F^{s}(X)-X=r \in \mathbb{Z}$, then $\rho_{F}(X)=\lim _{n \rightarrow \infty} \frac{F^{n}(X)-X}{n}=r / s$. Also, if $Y$ belongs to the same cycle as $X$ then $\rho(X)=\rho(Y)$. Thus, if $P$ is a cycle $(\bmod .1)$, in the sequel we shall also talk about the rotation number of $P$.

The set of all rotation numbers of $F$ will be denoted by $L_{F}$. From [6] it follows that $L_{F}$ is a closed interval on $\mathbb{R}$ (perhaps, degenerated to a single point). Thus, from now on $L_{F}$ will be called the rotation interval of $F$.

In what follows we shall denote by $\mathbb{N}$ the set of all positive integers. If $r \in \mathbb{Z}$ and $s \in \mathbb{N}$ are coprime and $s>1$ then there exist numbers $r_{1}, r_{2} \in \mathbb{Z}$ and $s_{1}, s_{2} \in \mathbb{N}$ such that

$$
r=r_{1}+r_{2}, \quad s=s_{1}+s_{2}, \text { and } r s_{1}-r_{1} s=r_{2} s-r s_{2}=1 .
$$

Clearly, these numbers are unique and, by (1), we have $r_{1} / s_{1}<r / s<r_{2} / s_{2}$. The interval $\left[r_{1} / s_{1}, r_{2} / s_{2}\right]$ is called the Farey interval for $r / s$.

In the above situation we define the two functions $\alpha_{r / s}: \mathbb{Z}^{+} \longrightarrow\left[r_{1} / s_{1}, r / s\right)$ and $\beta_{r / s}: \mathbb{Z}^{+} \longrightarrow\left(r / s, r_{2} / s_{2}\right]$ by

$$
\alpha_{r / s}(t)=\frac{r_{1}+t r}{s_{1}+t s} \text { and } \beta_{r / s}(t)=\frac{r_{2}+t r}{s_{2}+t s} .
$$


Now we can state our main result.

Theorem A. Let $r \in \mathbb{Z}, s, n \in \mathbb{N}$ be such that $r$ and $s$ are coprime. Assume that $F \in \mathcal{L}$ has a badly ordered cycle (mod. 1) of period ns and rotation number $r / s$. Then the rotation interval of $F$ contains an interval of the form $\left[\alpha_{r / s}(m), \beta_{r / s}(k)\right]$ for some non-negative integers $m, k$ with $m+k=n-1$ if $s>1$ and of the form $\left[r-\frac{1}{m}, r+\frac{1}{k}\right]$ for some positive integers $m, k$ with $m+k=n$ if $s=1$.

It is known (see for instance [1]) how to obtain the set of periods and lower bounds for the topological entropy of a map $F \in \mathcal{L}$ from the rotation interval of $F$. Thus, one can obtain via Theorem A this type of information, knowing only that a map has a badly ordered cycle of a given rotation number.

Theorem A in the case $n=1$ was proved by Boyland and Hall in [4] (see also Theorem 3.8.5 of [1]) and, for annulus homeomorphisms, by Boyland in [3]. Moreover, in Proposition 3.8.6 of [1] it was proved that the estimate of the rotation interval given by Theorem $\mathrm{A}$ in the case $n=1$ is the best possible. The next theorem extends this result to the general case.

Theorem B. Given $s, n \in \mathbb{N}$ and $r \in \mathbb{Z}$ with $r$ and $s$ coprime, and either non-negative integers $m, k$ with $m+k=n-1$ if $s>1$, or positive integers $m, k$ with $m+k=n$ if $s=1$, there exists a map $F \in \mathcal{L}$ having a badly ordered cycle (mod. 1) $P$ of period ns and rotation number $r / s$, such that the rotation interval of $F$ is either $\left[\alpha_{r / s}(m), \beta_{r / s}(k)\right]$ if $s>1$ or $\left[r-\frac{1}{m}, r+\frac{1}{k}\right]$ if $s=1$.

Remark 1.1. Notice that Theorem B does not cover the case $s=1, n=1$. Fortunately, there are no badly ordered cycles (mod. 1) of period 1.

The following result is a kind of converse of Theorem A.

Theorem C. Let $r \in \mathbb{Z}, s, n \in \mathbb{N}$ be such that $r$ and $s$ are coprime. Assume that the rotation interval of a map $F \in \mathcal{L}$ contains in its interior an interval of the form $\left[\alpha_{r / s}(m), \beta_{r / s}(k)\right]$ for some non-negative integers $m, k$ with $m+k=n-1$ if $s>1$ and of the form $\left[r-\frac{1}{m}, r+\frac{1}{k}\right]$ for some positive integers $m, k$ with $m+k=n$ if $s=1$. Then $F$ has a badly ordered cycle (mod. 1) of period $n s$ and rotation number $r / s$.

Theorem $\mathrm{C}$ is not the exact converse of Theorem A, since we assume that the appropriate interval is contained in the interior of the rotation interval of $F$. This cannot be helped, as the following example shows. The rotation interval corresponding to a badly ordered cycle (mod. 1) of period 2 and rotation number $1 / 2$ is $[0,1]$. Such a cycle (mod. 1 ) is badly ordered if and 
only if the map moves some of its points to the left. However, for every $b>0$ there exists a map in $\mathcal{L}$ with rotation interval $[0, b]$ that does not move any point to the left. Hence, even for very large $b$, there will be no badly ordered cycle (mod. 1) of period 2 and rotation number $1 / 2$.

From Theorems $\mathrm{A}$ and $\mathrm{C}$ we immediately get the following two corollaries which characterize the endpoints of the rotation interval of a map from $\mathcal{L}$ in terms of the behavior of the corresponding cycles (mod. 1). Let $A$ be a subset of $\mathbb{R}$. As usual $\operatorname{Bd}(A)$ will denote the boundary of $A$.

Corollary D. Let $a \in \mathbb{Q}$ and let $F \in \mathcal{L}$. Assume that $a \in L_{F}$. Then $a \in \operatorname{Bd}\left(L_{F}\right)$ if and only if each cycle (mod. 1$)$ of $F$ with rotation number a has a block structure over a twist cycle (mod. 1) of rotation number a.

The "if" part of Corollary D follows from Theorem C and the "only if" part from Theorem A.

Corollary E. Let $a \in \mathbb{Q}$ and let $F \in \mathcal{L}$. Assume that $a \in L_{F}$. Then $L_{F}=\{a\}$ if and only if each cycle (mod. 1) of F has a block structure over a twist cycle (mod. 1) of rotation number a.

The "if" part of Corollary E is trivial and the "only if" part follows from Corollary D.

To complete the picture, we notice that from Lemma 4.9 it follows that if a cycle (mod. 1) is not badly ordered then there exists a map $F \in \mathcal{L}$ with this cycle such that $L_{F}$ consists of one point. From this and Corollary E we can get Theorem 3.12 .20 of [1].

Theorem A and Corollary E improve results of MacKay and Tresser (see [7]). Corollary D for cycles having period equal to the denominator of $a$ (with $a$ written in irreducible form) has been proved in [2].

The paper is organized as follows. The proof of Theorem A is divided into two parts, dynamical (Section 2) and number theoretical (Section 3). Theorem B is proved in Section 4. Lastly, Theorem C is proved in Section 5.

Acknowledgements. The authors thank Phil Boyland and John Guaschi for calling their attention to this problem and useful discussions. All authors have been partially supported by the DGICYT grant number PB900695, and the third author by the NSF grant DMS-9305899. This paper has been made possible by an invitation of the third author by the Universitat Autònoma de Barcelona.

\section{Dynamical part of Theorem A.}

The goal of this section is to prove the following result which is the dynamical part of the proof of Theorem A. 
Proposition 2.1. Let $F \in \mathcal{L}, r \in Z$ and $s, n \in \mathbb{N}$ be such that $r$ and $s$ are coprime. Assume that $F$ has a badly ordered cycle of period ns and rotation number $r / s$. Then, there exist $a, c \in \mathbb{Z}$ and $b, d \in \mathbb{N}$ such that $a+c=n r$, $b+d=n s, a / b \neq r / s \neq c / d$ and both $a / b$ and $c / d$ belong to $L_{F}$.

Standing Hypotheses. In the rest of this section we will assume that $P$ is a badly ordered cycle of a map $F \in \mathcal{L}$ of period ns and rotation number $r / s$ with $n \in \mathbb{N}$ and $r$ and $s$ coprime.

Let $f$ denote the circle map of degree one which has $F$ as a lifting and set $G=F^{s}-r \in \mathcal{L}$. It is not difficult to see that $G$ is a lifting of $f^{s}$, the cycle $e(P)$ of $f$ decomposes into $s$ cycles $\widetilde{P}_{1}, \widetilde{P}_{2}, \ldots, \widetilde{P}_{s}$ of $f^{s}$ of period $n$ each. Moreover, $e^{-1}\left(\widetilde{P}_{i}\right)$ is a cycle (mod. 1) of $G$ of rotation number 0 for each $i \in\{1,2, \ldots, s\}$. So, for each $i \in\{1,2, \ldots, s\}$, we have that $e^{-1}\left(\widetilde{P}_{i}\right)$ decomposes into countable many cycles of $G$ of period $n$. Hence, $P$ is a countable union of cycles of $G$ of period $n$ each. We shall denote the set of all these cycles by $\Lambda$.

If $Q \in \Lambda$ then we shall say that $X, Y \in Q$ belong to the same component of $Q$ if every $Z \in P$ which lies between $X$ and $Y$ belongs to $Q$. Thus, every element of $\Lambda$ is divided into components. Notice that each element of $\Lambda$ has only one component if and only if $P$ has a block structure over a cycle of period $s$.

Before proving Proposition 2.1 we need a technical lemma and some more notation.

Lemma 2.2. There exist $X, Y \in P$ belonging to different cycles of $G$ such that $X<Y$ and $F(X)>F(Y)$.

Proof. Consider first the case when $P$ has a block structure over a cycle (mod. 1) $R$ of $F$ of period $s$. Since $P$ is badly ordered, $R$ is not twist. Thus, there are points $\tilde{X}, \tilde{Y} \in R$ with $\tilde{X}<\tilde{Y}$ such that $F(\tilde{X})>F(\tilde{Y})$. We can take $X$ and $Y$ from the blocks of $P$ corresponding to $\widetilde{X}$ and $\widetilde{Y}$ respectively. Then $X<Y$ and $F(X)>F(Y)$. Since the blocks of $P$ coincide with the elements of $\Lambda$, the proof is complete in this case.

Now we consider the case when $P$ has no block structure over any cycle (mod. 1) of $F$ of period $s$. Then there is an element $Q$ of $\Lambda$ with at least two components. Suppose that whenever $X, Y \in P$ belong to different cycles of $G$ and $X<Y$ then $F(X)<F(Y)$ (we cannot have $F(X)=F(Y)$ since $P$ is a cycle) and we will arrive to a contradiction. Take $X$ and $Z$ from different components of $Q$ such that $X<Z$. Then there is $Y \in P$ such that $X<Y<Z$ and $Y \notin Q$. We have $F(X)<F(Y)<F(Z)$. Since $F$ is a bijection of $P$ onto itself and commutes with $G$, the points $F(X)$ 
and $F(Z)$ belong to the cycle $F(Q) \in \Lambda$ and $F(Y)$ belongs to a different element of $\Lambda$. Thus, we can continue the same procedure and after $s$ steps we get $F^{s}(X)<F^{s}(Y)<F^{s}(Z)$. Therefore $G(X)<G(Y)<G(Z)$. Hence, $G(X)$ and $G(Z)$ belong to different components of $Q$ and $G(X)<G(Z)$. So, if we choose one point from every component of $Q$ in such a way that $X_{1}<X_{2}<\ldots<X_{j}$ then $G\left(X_{1}\right)<G\left(X_{2}\right)<\ldots<G\left(X_{j}\right)$ and each of the points $G\left(X_{i}\right)$ belongs to a different component of $Q$. Thus for each $i$ the points $X_{i}$ and $G\left(X_{i}\right)$ belong to the same component of $Q$. This proves that each component of $Q$ is mapped by $G$ into itself. Since $Q$ has more than one component and is a cycle of $G$, we get a contradiction. This completes the proof of the lemma.

Let $F \in \mathcal{L}$. We define

$$
\begin{aligned}
F_{l}(X) & =\inf \{F(Y): Y \geq X\}, \quad \text { and } \\
F_{u}(X) & =\sup \{F(Y): Y \leq X\} .
\end{aligned}
$$

It is not difficult to see that $F_{l}, F_{u} \in \mathcal{L}$, are non-decreasing and that $F_{l} \leq F \leq$ $F_{u}$. It is well known (see for instance [1]) that if $G \in \mathcal{L}$ is non-decreasing, then

$$
\lim _{n \rightarrow \infty} \frac{G^{n}(X)-X}{n}
$$

exists for each $X \in \mathbb{R}$ and is independent of $X$. This number is called the rotation number of $G$ and denoted by $\rho(G)$. Also it is well known that if $F \in \mathcal{L}$ then $L_{F}=\left[\rho\left(F_{l}\right), \rho\left(F_{u}\right)\right]$ (see [1]).

Proof of Proposition 2.1. Let $X$ and $Y$ be the points from the statement of Lemma 2.2. We define a map $\Phi: P \rightarrow P$ as follows. For all $k \in \mathbb{Z}$ we set $\Phi(X+k)=F(Y+k)$ and $\Phi(Y+k)=F(X+k)$. For all other points $Z \in P$ we set $\Phi(Z)=F(Z)$. Notice that for every $T \in P$ and $k \in \mathbb{Z}$ we have $\Phi(T+k)=\Phi(T)+k$, so $\Phi$ is a restriction to $P$ of a lifting $\widetilde{\Phi}$ of some degree one map $\varphi: \mathbb{S}^{1} \rightarrow \mathbb{S}^{1}$. For $\varphi$ the set $e(P)$ is the union of two cycles.

We have $Y=F^{b}(X)-a$ for some integers $a$ and $b$ with $0 \leq b \leq n s-1$. However, $F(X-a)=F(X)-a$, so the signs of $X-(X-a)$ and $F(X)-$ $F(X-a)$ are the same. Therefore, by the choice of $X$ and $Y, b$ cannot be 0 . Thus, $1 \leq b \leq n s-1$. We have $\Phi^{i}(Y)=F^{i}(X)$ for $i=1,2, \ldots, b$. Therefore the rotation number of $Y$ for $\widetilde{\Phi}$ is $\left(F^{b}(X)-Y\right) / b=a / b$. Similarly, we have $\Phi^{i}(X)=F^{b+i}(X)-a$ for $i=1,2, \ldots, n s-b$. Therefore the rotation number of $X$ for $\widetilde{\Phi}$ is $\left(F^{n s}(X)-a-X\right) /(n s-b)=(n r-a) /(n s-b)$. We set $c=n r-a$ and $d=n s-b$, and then the rotation number of $X$ is $c / d$. By the definition, we have $a+c=n r$ and $b+d=n s$.

We claim that $F_{l}(Z) \leq \Phi(Z) \leq F_{u}(Z)$ for all $Z \in P$. This is clearly true if $Z$ is not of the form $X+k$ or $Y+k$ for $k \in \mathbb{Z}$, since then $\Phi(Z)=F(Z)$. 
If $Z=X+k$ then, since $Y+k>X+k$, we have $F_{l}(Z)=F_{l}(X+k) \leq$ $F_{l}(Y+k) \leq F(Y+k)=\Phi(X+k)=\Phi(Z)$. Since $F(X)>F(Y)$, we get $\Phi(Z)=\Phi(X+k)=F(Y+k)=F(Y)+k<F(X)+k=F(X+k) \leq$ $F_{u}(X+k)=F_{u}(Z)$. If $Z=Y+k$ then we get $F_{l}(Z) \leq \Phi(Z) \leq F_{u}(Z)$ in a similar way.

Since $F_{l}$ is non-decreasing and $\left.F_{l}\right|_{P} \leq \Phi$, the rotation number of $F_{l}$ is smaller than or equal to the rotation numbers of $X$ and $Y$ for $\widetilde{\Phi}$. That is, $\rho\left(F_{l}\right) \leq a / b$ and $\rho\left(F_{l}\right) \leq c / d$. Analogously, $\rho\left(F_{u}\right) \geq a / b$ and $\rho\left(F_{u}\right) \geq c / d$. Since the rotation interval of $F$ is $\left[\rho\left(F_{l}\right), \rho\left(F_{u}\right)\right]$, we see that both $a / b$ and $c / d$ belong to it.

It remains to prove that $a / b \neq r / s \neq c / d$. In fact, it suffices to prove the first inequality, since if $c / d=r / s$ then $a / b=(n r-c) /(n s-d)=r / s$.

Suppose that $a / b=r / s$. Since $r$ and $s$ are coprime, we see that $s$ divides $b$. Therefore, since $X$ and $Y$ belong to different cycles of $G$ we have that $F^{b}(X)-r b / s=G^{b / s}(X) \neq Y$. Hence $a / b=\left(F^{b}(X)-Y\right) / b \neq r / s$. This completes the proof.

\section{Number theoretical part of Theorem A.}

In the next lemma and corollary we assume that $n, s, s_{1}, s_{2}, b, d \in \mathbb{N}$ and $r, r_{1}, r_{2}, a, c \in \mathbb{Z}$ are such that $r$ and $s$ are coprime, $s>1,\left[r_{1} / s_{1}, r_{2} / s_{2}\right]$ is the Farey interval for $r / s$ (that is, (1) is satisfied), $a / b<r / s<c / d$, and

$$
a+c=n r \text { and } b+d=n s .
$$

The functions $\alpha_{r / s}$ and $\beta_{r / s}$ are defined as in Section 1.

By (1) we have $\alpha_{r / s}^{\prime}(t)=\left(r s_{1}-r_{1} s\right) /\left(s_{1}+t s\right)^{2}>0$. Thus, $\alpha_{r / s}$ is increasing. By (1), $r_{1}+\left(-s_{1} / s\right) r<0$, so $\lim _{t \searrow-s_{1} / s} \alpha_{r / s}(t)=-\infty$. Clearly, $\lim _{t \rightarrow+\infty} \alpha_{r / s}(t)=r / s$. Similarly, $\beta_{r / s}$ is decreasing, $\lim _{t \searrow-s_{2} / s} \beta_{r / s}(t)=+\infty$, and $\lim _{t \rightarrow+\infty} \beta_{r / s}(t)=r / s$.

Lemma 3.1. There exist non-negative integers $m, k$ such that $m+k \leq n-1$ and $a / b \leq \alpha_{r / s}(m), c / d \geq \beta_{r / s}(k)$.

Proof. Since $a / b<r / s$, there is a unique $\gamma \geq-s_{1} / s$ such that $\alpha_{r / s}(\gamma)=a / b$. Simple computation gives $\gamma=\left(a s_{1}-b r_{1}\right) /(b r-a s)$. Similarly, there is a unique $\delta \geq-s_{2} / s$ such that $\beta_{r / s}(\delta)=c / d$ and we get $\delta=\left(d r_{2}-c s_{2}\right) /(c s-$ $d r)$. Notice that $b r-a s$ and $c s-d r$ are positive, and since they are integers, they are larger than or equal to 1. Moreover, by (1) and (2) we get

$$
\begin{aligned}
a s_{1}-b r_{1} & =(n r-c) s_{1}-(n s-d) r_{1} \\
& =n\left(r s_{1}-s r_{1}\right)-\left(c s_{1}-d r_{1}\right)=n-\left(c s_{1}-d r_{1}\right) .
\end{aligned}
$$


Assume first that $\gamma$ and $\delta$ are non-negative. Then we set $m=a s_{1}-b r_{1}$ and $k=d r_{2}-c s_{2}$. Then $m \geq \gamma$ and $k \geq \delta$, so $a / b=\alpha_{r / s}(\gamma) \leq \alpha_{r / s}(m)$ and $c / d=\beta_{r / s}(\delta) \geq \beta_{r / s}(k)$. By (3) and (1) we get

$$
\begin{aligned}
m+k & =n-c s_{1}+d r_{1}+d r_{2}-c s_{2} \\
& =n-c\left(s_{1}+s_{2}\right)+d\left(r_{1}+r_{2}\right)=n-(c s-d r) \leq n-1 .
\end{aligned}
$$

If $d r_{2}-c s_{2}<0$ then we set $m=n-1$ and $k=0$. Then clearly $c / d>$ $r_{2} / s_{2}=\beta_{r / s}(0)=\beta_{r / s}(k)$. Since $r_{1} / s_{1}<r / s<c / d$, we have $c s_{1}-d r_{1} \geq 1$, so by (3), $a s_{1}-b r_{1} \leq n-1$. Therefore $\gamma \leq n-1$. Thus, $a / b=\alpha_{r / s}(\gamma) \leq$ $\alpha_{r / s}(n-1)=\alpha_{r / s}(m)$.

If $a s_{1}-b r_{1}<0$ then we set $m=0$ and $k=n-1$. In a similar way as above we get $a / b<\alpha_{r / s}(m)$ and $c / d \geq \beta_{r / s}(k)$.

Since $\alpha_{r / s}$ is increasing and $\beta_{r / s}$ is decreasing, we obtain immediately the following corollary.

Corollary 3.2. There exist non-negative integers $m, k$ such that $m+k=$ $n-1, a / b \leq \alpha_{r / s}(m)$ and $c / d \geq \beta_{r / s}(k)$.

Now we assume that $s=1$. Then there is no Farey interval for $r / s$. In this case we get the following simple result.

Lemma 3.3. If $a / b, c / d$ and $r / s$ are as above and $s=1$ then $a / b \leq$ $(b r-1) / b<r<(d r+1) / d \leq c / d$.

Proof. Since $a / b<r$, we get $a<b r$, so $a \leq b r-1$. Similarly, $c \geq d r+$ 1.

Proof of Theorem A. By Proposition 2.1, we get $a, c \in \mathbb{Z}$ and $b, d \in \mathbb{N}$ such that $a+c=n r, b+d=n s, a / b \neq r / s \neq c / d$ and both $a / b$ and $c / d$ belong to the rotation interval of $F$. If $a / b>r / s$ then $c / d=(n r-a) /(n s-b)<r / s$, so without loss of generality we may assume that $a / b<r / s$. Then $c / d>r / s$ and we use Corollary 3.2 if $s>1$ and Lemma 3.3 if $s=1$.

\section{Proof of Theorem B.}

We start by choosing $r \in \mathbb{Z}$ and $n, s \in \mathbb{N}$ such that $r$ and $s$ are coprime. Then, we take a set $P=\{i /(n s): i \in \mathbb{Z}\} \subset \mathbb{R}$. To prove Theorem B we want to make $P$ a badly ordered cycle of a map from $\mathcal{L}$ with the appropriate rotation interval. If $n=1$ then this is done in Proposition 3.8.6 of [1]. 
Therefore we assume $n>1$. We start by defining two maps $\Psi_{0}, \Psi_{1}: P \rightarrow P$ in several steps.

Let $1 \leq u \leq n-1$. Then we define a permutation $\zeta$ of the set $\{0,1, \ldots, n-$ $1\}$ as follows:

$$
\zeta(j)= \begin{cases}j+1 & \text { if } 0 \leq j \leq u-2 \\ n-1 & \text { if } j=u-1 \\ 0 & \text { if } j=u \\ j-1 & \text { if } u+1 \leq j \leq n-1\end{cases}
$$

It is not difficult to see that $\zeta$ is a cyclic permutation.

Next we define a map $\Xi: P \rightarrow P$ as follows. For each $k \in \mathbb{Z}$ we set

$$
\Xi\left(\frac{i}{n s}+k\right)=\left\{\begin{array}{cl}
\frac{\zeta(i)}{n s}+k+\frac{r}{s} & \text { if } i \in\{0,1, \ldots, n-1\}, \\
\frac{i}{n s}+k+\frac{r}{s} & \text { otherwise. }
\end{array}\right.
$$

Remark 4.1. For each $k \in \mathbb{Z}$ and $X \in P$ we have that $\Xi(X+k)=$ $\Xi(X)+k$.

Let $P \subset \mathbb{R}$ be such that $P \cap[0,1)$ is finite and $X+m \in P$ for all $X \in P$ and $m \in \mathbb{Z}$. Let $\Gamma: P \rightarrow P$ be a map such that $\Gamma(X+1)=\Gamma(X)+1$ for each $X \in P$. A map $F \in \mathcal{L}$ will be called $(P, \Gamma)$-linear if $\left.F\right|_{P}=\left.\Gamma\right|_{P}$ and $F$ is affine on each interval $[X, Y]$ such that $X, Y \in P$ and $(X, Y) \cap P=\emptyset$. We note that we have defined $\Xi$ in such a way that the set $P$ is a cycle (mod. 1$)$ of the $(P, \Xi)$-linear map, of period $n s$ which has a block structure over a twist cycle of rotation number $r / s$. Moreover, it also has rotation number $r / s$, as the next simple lemma shows.

For a set $A \subset \mathbb{R}$ and $X \in \mathbb{R}$ we will denote by $X+A$ or $A+X$ the set $\{X+Y: Y \in A\}$.

Lemma 4.2. Let $P$ and $Q$ be cycles (mod. 1) of the maps $F$ and $G$ from $\mathcal{L}$ respectively. Assume that $P$ has a block structure over $Q$. Then $\rho_{F}(P)=$ $\rho_{G}(Q)$.

Proof. Assume that $Q$ has period $s$ and rotation number $r / s$, and $P$ has period ns. Let $X \in P$ belong to a block $R$ corresponding to $Y \in Q$. Then $F^{n s}(X)$ belongs to the block corresponding to $G^{n s}(Y)=Y+n r$. Therefore $F^{n s}(X) \in R+n r$. Since the diameter of any block is smaller than 1 and $F^{n s}(X)-X$ is an integer, we get $F^{n s}(X)=X+n r$. Therefore $\rho_{F}(X)=$ $r / s$. 
To define the maps we are looking for we first define the switch $\Sigma_{X}: P \longrightarrow P$ at a point $X \in P$ as follows:

$$
\Sigma_{X}(Y)= \begin{cases}Y-1 /(n s) & \text { if } Y=X+k, k \in \mathbb{Z}, \\ Y+1 /(n s) & \text { if } Y=X+k-1 /(n s), k \in \mathbb{Z}, \\ Y & \text { otherwise }\end{cases}
$$

Notice that $\Sigma_{X}^{-1}=\Sigma_{X}$.

Now we set $\Psi_{0}=\Sigma_{0} \circ \Xi \circ \Sigma_{0}$ and $\Psi_{1}=\Sigma_{1 / s} \circ \Xi \circ \Sigma_{1 / s}$.

Remark 4.3. For each $k \in \mathbb{Z}$ and $X, Y \in P$ we have $\Sigma_{X}(Y+k)=\Sigma_{X}(Y)+$ $k$. Therefore, for each $k \in \mathbb{Z}$ and $Y \in P$ we have $\Psi_{0}(Y+k)=\Psi_{0}(Y)+k$ and $\Psi_{1}(Y+k)=\Psi_{1}(Y)+k$. Hence the $\left(P, \Psi_{0}\right)$-linear and $\left(P, \Psi_{1}\right)$-linear maps belong to $\mathcal{L}$.

Lemma 4.4. The set $P$ is a cycle (mod. 1) of the $\left(P, \Psi_{0}\right)$-linear and $\left(P, \Psi_{1}\right)$-linear maps with period $n s$ and rotation number $r / s$.

Proof. We will prove that $P$ is a cycle (mod. 1$)$ of the $\left(P, \Psi_{0}\right)$-linear map with period $n s$ and rotation number $r / s$. The other statement follows similarly.

Let us look for the smallest $m \in \mathbb{N}$ such that $\Psi_{0}^{m}(X)=X+k$ for some $k \in \mathbb{Z}$, where $X=-1 /(n s)$. Since $\Psi_{0}^{m}=\Sigma_{0} \circ \Xi^{m} \circ \Sigma_{0}$ (recall that $\Sigma_{0}^{-1}=\Sigma_{0}$ ), $\Sigma_{0}(X)=0$, and $\Sigma_{0}^{-1}(X+k)=k$, the equation $\Psi_{0}^{m}(X)=X+k$ is equivalent to the equation $\Xi^{m}(0)=k$. We have

$$
\Xi^{m}(0)=\frac{\zeta^{l}(0)}{n s}+m \frac{r}{s}
$$

where $l$ is the integer part of $(m+s-1) / s$. Thus our equation becomes

$$
\frac{\zeta^{l}(0)}{n s}+\frac{m r}{s}=k
$$

Since $0 \leq \zeta^{l}(0)<n$, the above equation has a solution if and only if $\zeta^{l}(0)=0$. Since $\zeta$ is a cyclic permutation on $n$ elements, this means that $l=n q$ for some integer $q$. The equation is now $\mathrm{mr} / \mathrm{s}=k$. Since $r$ and $s$ are coprime, we get $m=p s$ for some integer $p$. Since $(m+s-1) / s=p+(s-1) / s$, we have $l=p$, and therefore $m=q n s$. The smallest positive $m$ with this property is $m=n s$. Since $X \in P$ and the cardinality of $P \cap[0,1)$ is $n s$, this means that $P$ is a cycle (mod. 1$)$ of the $\left(P, \Psi_{0}\right)$-linear map with period $n s$. We have $\Psi_{0}^{n s}(X)=n s r / s=n r$, so $\rho_{\Psi_{0}}(P)=r / s$.

In the rest of the section we will denote $n-u$ by $l$ and we will use freely the notation from the introduction.

Proposition 4.5. Assume that $s>1$. Then, the rotation interval of the $\left(P, \Psi_{0}\right)$-linear map is $\left[\alpha_{r / s}(l), \beta_{r / s}(u-1)\right]$. Similarly, the rotation interval of the $\left(P, \Psi_{1}\right)$-linear map is $\left[\alpha_{r / s}(l-1), \beta_{r / s}(u)\right]$. 
To prove Proposition 4.5, we need two auxiliary lemmas.

Lemma 4.6. Let $\widetilde{\Psi}_{0}$ be defined as $\Psi_{0}$, but with $u$ and $l$ interchanged and $r$ replaced by $-r$. Then the rotation interval of the $\left(P, \Psi_{1}\right)$-linear map is equal to minus the rotation interval of the $\left(P, \widetilde{\Psi}_{0}\right)$-linear map (that is, if the rotation interval of the $\left(P, \widetilde{\Psi}_{0}\right)$-linear map is $[x, y]$ then the rotation interval of the $\left(P, \Psi_{1}\right)$-linear map is $\left.[-y,-x]\right)$.

Proof. Let $\Delta$ be a homeomorphism of the real line given by $\Delta(X)=(n-$ $1) /(n s)-X$. Notice that $\Delta^{2}$ is the identity. If the permutation $\widetilde{\zeta}$ is like $\zeta$, but with $u$ and $l$ interchanged, then $\widetilde{\zeta}=\Delta_{1} \circ \zeta \circ \Delta_{1}$, where $\Delta_{1}(i)=$ $n-1-i$. Therefore if the map $\widetilde{\Xi}$ is like $\Xi$, but with $u$ and $l$ interchanged and $r$ replaced by $-r$ then $\widetilde{\Xi}=\Delta \circ \Xi \circ \Delta$. Furthermore, $\Delta \circ \Sigma_{0} \circ \Delta=\Sigma_{1 / s}$. Since $\widetilde{\Psi}_{0}=\Sigma_{0} \circ \widetilde{\Xi} \circ \Sigma_{0}$, we get $\Delta \circ \widetilde{\Psi}_{0} \circ \Delta=\Psi_{1}$. Therefore for any $k$ we have $\Delta \circ \widetilde{\Psi}_{0}^{k} \circ \Delta=\Psi_{1}^{k}$, so

$$
\frac{\Psi_{1}^{k}(X)-X}{k}=\frac{\Delta\left(\left(\widetilde{\Psi}_{0}^{k} \circ \Delta\right)(X)\right)-\Delta(\Delta(X))}{k}=-\frac{\widetilde{\Psi}_{0}^{k}(\Delta(X))-\Delta(X)}{k} .
$$

When we take the limit superior as $k \rightarrow \infty$ of both sides, and then the minimum (respectively maximum) over all $X \in P$ then we get at the left hand side the left (respectively the right) endpoint of the rotation interval of the $\left(P, \Psi_{1}\right)$-linear map, and at the right hand side minus the right (respectively minus the left) endpoint of the $\left(P, \widetilde{\Psi}_{0}\right)$-linear map.

Lemma 4.7. The following conditions are equivalent:

(i) $\frac{n-1}{n s}+\frac{r}{s}+(u-1) r=-\frac{1}{n s}+k$ for some $k \in \mathbb{Z}$,

(ii) $s \mid(r+1)$,

(iii) $s_{2}=1$.

Proof. Condition (i) is equivalent to $n-1+n r \equiv-1(\bmod n s)$ which is equivalent to $1+r \equiv 0(\bmod s)$ which, in turn, is equivalent to (ii). By (1), (ii) is equivalent to (iii).

Proof of Proposition 4.5. Let $F$ be the $\left(P, \Psi_{0}\right)$-linear map. Let us describe the maps $F_{u}$ and $F_{l}$ restricted to $P$. For $k \in \mathbb{Z}$ we have:

$$
F_{u}\left(\frac{i}{n s}+k\right)= \begin{cases}k & \text { if } i=-n r-1, \\ \frac{1}{n s}+\frac{r}{s}+k & \text { if } i=-1, \\ \frac{i+1}{n s}+\frac{r}{s}+k & \text { if } 0 \leq i \leq u-2, \\ \frac{n-1}{n s}+\frac{r}{s}+k & \text { if } u-1 \leq i \leq n-2, \\ \frac{i}{n s}+\frac{r}{s}+k & \text { otherwise. }\end{cases}
$$


Similarly,

$$
F_{l}\left(\frac{i}{n s}+k\right)= \begin{cases}\frac{-1}{n s}+k & \text { if } i=-n r \\ \frac{-1}{n s}+\frac{r}{s}+k & \text { if } i=0 \\ \frac{r}{s}+k & \text { if } 1 \leq i \leq u \\ \frac{i-1}{n s}+\frac{r}{s}+k & \text { if } u+1 \leq i \leq n-1, \\ \frac{i}{n s}+\frac{r}{s}+k & \text { otherwise. }\end{cases}
$$

Now we define the sets $P_{0}, P_{u}$ and $P_{l}$ as follows:

$$
\begin{aligned}
P_{0} & =\{i /(n s)+k: 0 \leq i \leq n-1, k \in \mathbb{Z}\}, \\
P_{u} & =\{i /(n s): i \equiv-1(\bmod n)\}, \\
P_{l} & =\{i /(n s): n \mid i\} .
\end{aligned}
$$

In order to compute the rotation number of the map $F_{u}$ let us investigate this map closer. If $X=i /(n s)+k$ with $0 \leq i \leq u-2$ then $F_{u}^{j}(X) \notin P_{0} \cup P_{u}$ for $j=1,2, \ldots, s-1$, but $F_{u}^{s}(X) \in P_{0}$. We get then $F_{u}^{s}(X)=(i+1) /(n s)+r+k$. Therefore $F_{u}^{(u-1) s}(0)=(u-1) /(n s)+(u-1) r$. Hence

$$
F_{u}^{(u-1) s+1}(0)=\frac{n-1}{n s}+\frac{r}{s}+(u-1) r .
$$

This point belongs to $P_{u}$ and its images stay in $P_{u}$ until some of them becomes of the form $(-n r-1) /(n s)+k$ or $-1 /(n s)+k$. In the meantime we just add $r / s$ to get the next image. Since $-1 /(n s)+k=(-n r-1) /(n s)+$ $k+r / s$, we arrive to $(-n r-1) /(n s)+k$ first, unless already

$$
\frac{n-1}{n s}+\frac{r}{s}+(u-1) r=-\frac{1}{n s}+k .
$$

Suppose that (5) does not hold. Then we get to $(-n r-1) /(n s)+k$ from $(n-1) /(n s)+r / s+(u-1) r$ in $m$ steps, where

$$
\frac{-n r-1}{n s}+k=\frac{n-1}{n s}+\frac{r}{s}+(u-1) r+\frac{m r}{s},
$$

and $m$ is the smallest non-negative integer satisfying (6) for some $k \in \mathbb{Z}$. This condition is equivalent to $-n r-1 \equiv n-1+n r+m n r(\bmod n s)$, which is equivalent to $n+2 n r+m n r \equiv 0(\bmod n s)$, which, in turn, is equivalent to $1+(2+m) r \equiv 0(\bmod s)$. This gives us $m+2=s_{2}$, unless $s_{2}=1$. However, by Lemma 4.7, $s_{2}=1$ is equivalent to (5), and we assumed that (5) does not hold. Therefore $m=s_{2}-2$. 
After one more step we get to $k$. Thus, in view of $(4)$, it takes $((u-1) s+$ $1)+\left(s_{2}-2\right)+1=(u-1) s+s_{2}$ steps to get from 0 to $k$. Moreover, by (6) we get

$$
\begin{aligned}
k & =\frac{n-1}{n s}+\frac{r}{s}+(u-1) r+\frac{m r}{s}+\frac{n r+1}{n s} \\
& =\frac{n-1+n r+\left(s_{2}-2\right) r n+n r+1}{n s}+(u-1) r \\
& =\frac{1+s_{2} r}{s}+(u-1) r=r_{2}+(u-1) r .
\end{aligned}
$$

Therefore

$$
\rho\left(F_{u}\right)=\frac{(u-1) r+r_{2}}{(u-1) s+s_{2}}=\beta_{r / s}(u-1) .
$$

Suppose now that (5) holds. Then $F_{u}((n-1) /(n s)+r / s+(u-1) r)=$ $1 /(n s)+r / s+k$. Since $F_{u}(0)=1 /(n s)+r / s$, we get

$$
F_{u}^{(u-1) s}\left(\frac{1}{n s}+\frac{r}{s}\right)=\frac{n-1}{n s}+\frac{r}{s}+(u-1) r
$$

and, hence

$$
F_{u}^{(u-1) s+1}\left(\frac{1}{n s}+\frac{r}{s}\right)=\frac{1}{n s}+\frac{r}{s}+k,
$$

where $k=(n-1) /(n s)+r / s+(u-1) r+1 /(n s)=(n-1+n r+1) /(n s)+$ $(u-1) r=(r+1) / s+(u-1) r$. By Lemma 4.7 we have $s_{2}=1$, so $r_{2} s=r+1$. Therefore

$$
F_{u}^{(u-1) s+s_{2}}\left(\frac{1}{n s}+\frac{r}{s}\right)=\frac{1}{n s}+\frac{r}{s}+\left(r_{2}+(u-1) r\right) .
$$

Thus we obtain again (7).

Now we compute the rotation number of $F_{l}$. If $X=i /(n s)+k$ with $u+1 \leq i \leq n-1$ then $F_{l}^{j}(X) \notin P_{0} \cup P_{l}$ for $j=1,2, \ldots, s-1$, but $F_{l}^{s}(X) \in P_{0}$. We get then $F_{l}^{s}(X)=(i-1) /(n s)+r+k$. Therefore $F_{l}^{(l-1) s}((n-1) /(n s))=$ $u /(n s)+(l-1) r$. Then $F_{l}^{(l-1) s+1}((n-1) /(n s))=r / s+(l-1) r$. This point belongs to $P_{l}$ and its image stays in $P_{l}$ for the next $s-2$ steps, when we get $F_{l}^{l s-1}((n-1) /(n s))=r / s+(l-1) r+(s-2) r / s=l r-r / s$. After one more step we get $F_{l}^{l s}((n-1) /(n s))=l r-1 /(n s)$. Again we add $r / s$ in each step until, after $m$ steps, we reach $(n-1) /(n s)+k$ for some $k$. We have $(n-1) /(n s)+k=l r-1 /(n s)+m r / s$, and $m$ is the smallest non-negative integer satisfying this equality with some $k$. We get $n-1 \equiv$ 
$-1+m r n(\bmod n s)$, so $m r \equiv 1(\bmod s)$. Therefore $m=s_{1}$. Thus,

$$
\begin{aligned}
F_{l}^{l s+s_{1}}\left(\frac{n-1}{n s}\right) & =l r-\frac{1}{n s}+\frac{s_{1} r}{s}=l r+\frac{s_{1} r n-1}{n s} \\
& =l r+\frac{\left(s r_{1}+1\right) n-1}{n s}=l r+r_{1}+\frac{n-1}{n s} .
\end{aligned}
$$

Hence we get

$$
\rho\left(F_{l}\right)=\frac{l r+r_{1}}{l s+s_{1}}=\alpha_{r / s}(l) .
$$

This proves that the rotation interval of $F$ is $\left[\alpha_{r / s}(l), \beta_{r / s}(u-1)\right]$.

To find the rotation interval of the $\left(P, \Psi_{1}\right)$-linear map, we take $\widetilde{\Psi}_{0}$ as in Lemma 4.6. By the part of the proposition already proven, the rotation interval of the $\left(P, \widetilde{\Psi}_{0}\right)$-linear map is $\left[\alpha_{-r / s}(u), \beta_{-r / s}(l-1)\right]$. By Lemma 4.6, the rotation interval of the $\left(P, \Psi_{1}\right)$-linear map is $\left[-\beta_{-r / s}(l-1),-\alpha_{-r / s}(u)\right]$. One can easily check that the Farey interval for $(-r) / s$ is minus the Farey interval for $r / s$, that is $\left[\left(-r_{2}\right) / s_{2},\left(-r_{1}\right) / s_{1}\right]$. Therefore $-\beta_{-r / s}(l-1)=$ $-\left(-r_{1}-(l-1) r\right) /\left(s_{1}+(l-1) s\right)=\alpha_{r / s}(l-1)$ and $-\alpha_{-r / s}(u)=-\left(-r_{2}-\right.$ $u r) /\left(s_{2}+u s\right)=\beta_{r / s}(u)$. This completes the proof.

We also need to deal with the case $s=1$. Notice that then $\Sigma_{0}=\Sigma_{1 / s}$, so $\Psi_{1}=\Psi_{0}$.

Proposition 4.8. Let $s=1$. Then the rotation interval of the $\left(P, \Psi_{0}\right)$ linear map is $\left[r-\frac{1}{l}, r+\frac{1}{u}\right]$.

Proof. Let $F$ be the $\left(P, \Psi_{0}\right)$-linear map. Let us describe $F_{u}$ and $F_{l}$ restricted to $P$. We can assume that $r=0$; then at the end we will have to add $r$ to both endpoints of the rotation interval to get the result in the general case. For $k \in \mathbb{Z}$ we have:

$$
F_{u}\left(\frac{i}{n}+k\right)= \begin{cases}\frac{i+1}{n}+k & \text { if } 0 \leq i \leq u-2 \\ k+1 & \text { if } u-1 \leq i \leq n-2 \\ \frac{1}{n}+k+1 & \text { if } i=n-1\end{cases}
$$

We get $F_{u}(0)=1 / n, F_{u}(1 / n)=2 / n, \ldots, F_{u}((u-2) / n)=(u-1) / n$, and $F_{u}((u-1) / n)=1$. Therefore $\rho\left(F_{u}\right)=1 / u$.

By Lemma 4.6 and since $\Psi_{1}=\Psi_{0}$, we have $\rho\left(F_{l}\right)=-\rho\left(\widetilde{F}_{u}\right)$, where $\widetilde{F}$ is the $\left(P, \widetilde{\Psi}_{0}\right)$-linear map. Since $r=0=-r$, we have $\rho\left(\widetilde{F}_{u}\right)=1 / l$, and thus $\rho\left(F_{l}\right)=-1 / l$. 
The next result will allow us to prove that $P$ is a badly ordered cycle of the maps which are $\left(P, \Psi_{0}\right)$-linear and $\left(P, \Psi_{1}\right)$-linear.

Lemma 4.9. Let $a \in \mathbb{Q}$ and let $F \in \mathcal{L}$. Assume that $F$ has a cycle (mod. 1$)$ $P$ such that $F$ is $\left(P,\left.F\right|_{P}\right)$-linear and $P$ has a block structure over a twist cycle (mod. 1) of rotation number $a$. Then $L_{F}=\{a\}$.

Proof. Let $a=r / s$ with $r$ and $s$ coprime. Let $P_{i}$ be the blocks of the block structure of $P$ over a twist cycle (mod. 1), numbered in such a way that if $X \in P_{i}$ and $Y \in P_{j}$ with $i<j$ then $X<Y$. Then, since the block structure is over a twist cycle (mod. 1) of rotation number $r / s$, we have $P_{i}+1=P_{i+s}$ and $F\left(P_{i}\right)=P_{i+r}$.

Set $X_{i}=\min P_{i}$ and $Y_{i}=\max P_{i}$. Define $G$ on $Q=\left\{X_{i}: i \in \mathbb{Z}\right\} \cup\left\{Y_{i}\right.$ : $i \in \mathbb{Z}\}$ by $G\left(X_{i}\right)=G\left(Y_{i}\right)=Y_{i+r}$, and extend it to a $Q$-linear map. Clearly, $G \in \mathcal{L}$ and $G$ is non-decreasing. Therefore it has a unique rotation number. Since $\left\{Y_{i}: i \in \mathbb{Z}\right\}$ is a twist cycle $(\bmod .1)$ of $G$ of rotation number $r / s$, we have $\rho_{G}=r / s=a$. Since $F \leq G$ and $G$ is non-decreasing, the right-hand endpoint of $L_{F}$ is not larger than $\rho_{G}$ (see e.g. [1]), that is not larger than $a$.

Similarly, we can show that the left-hand endpoint of $L_{F}$ is not smaller than $a$. Therefore $L_{F}=\{a\}$.

Proof of Theorem B. It follows from Lemmas 4.4 and 4.9, Propositions 4.5 and 4.8 and Proposition 3.8.6 of [1] (for the case $n=1$ ).

\section{Proof of Theorem C.}

Before starting the proof of Theorem $\mathrm{C}$ we will introduce some notions and preliminary results. The following lemma is well known (see for instance $[1])$.

Lemma 5.1. Let $F \in \mathcal{L}$ and let $P$ be a twist cycle (mod. 1) of $F$ with rotation number $r / s$. Assume that $(r, s)=1$ and $P=\left\{X_{i}\right\}_{i \in \mathbb{Z}}$ with $X_{i}<X_{j}$ if and only if $i<j$. Then $P$ has period $s$ and $F\left(X_{i}\right)=X_{i+r}$ for each $i \in \mathbb{Z}$.

A set $P \subset \mathbb{R}$ such that $P \cap[0,1]$ is finite and has the property that $P+m=P$ for each $m \in \mathbb{Z}$ will be called finite (mod. 1).

Let $P$ be finite (mod. 1 ) and let $F \in \mathcal{L}$. An interval $I \subset \mathbb{R}$ will be called $P$-basic if it is the closure of a component of $\mathbb{R} \backslash P$. A sequence $\left(I_{0}, I_{1}, \ldots, I_{k}\right)$ of $P$-basic intervals such that $F\left(I_{i}\right) \supset I_{i+1}$ for $i=0,1, \ldots, k-1$ and $I_{k}=$ $I_{0}+m$ with $m \in \mathbb{Z}$ will be called a loop of length $k$. Given such a loop, 
from Lemma 1.2.7 of [1] we see that there exists a point $X \in I_{0}$ such that $F^{i}(X) \in I_{i}$ for $i=1,2, \ldots, k-1$ and $F^{k}(X)=X+m$.

Lemma 5.2. Assume that $a<p_{1} / q_{1}<p_{2} / q_{2}<b$, where $a, b \in \mathbb{Q}$, and let $F \in \mathcal{L}$ be such that $[a, b] \subset L_{F}$. Then $F$ has twist cycles (mod. 1) $P$ and $Q$ of rotation numbers $a$ and $b$ respectively, such that

(i) each map $G \in \mathcal{L}$ such that $\left.G\right|_{P \cup Q}=\left.F\right|_{P \cup Q}$ has a cycle (mod. 1) $R$ of $G$-rotation number $\left(p_{1}+p_{2}\right) /\left(q_{1}+q_{2}\right)$ such that there is a point $X \in R$ with the property that $G^{q_{2}}(X)>X+p_{2}$,

(ii) there is a map $\widetilde{F} \in \mathcal{L}$ with $\left.\widetilde{F}\right|_{P \cup Q}=\left.F\right|_{P \cup Q}$ and $L_{\widetilde{F}}=[a, b]$.

Proof. Consider the family $F_{\mu}$ defined by

$$
F_{\mu}=\left(\min \left(F, F_{l}+\mu\right)\right)_{u}
$$

for $0 \leq \mu \leq \mu_{1}=\sup _{x \in \mathbb{R}}\left(F-F_{l}\right)(x)$ (see [5] or [1]). It is not difficult to see that $F_{0}=F_{l}, F_{\mu_{1}}=F_{u}$ and, for each $0 \leq \mu \leq \mu_{1}, F_{\mu}$ belongs to $\mathcal{L}$, is nondecreasing, and on parts where it is not locally constant coincides with $F$. Therefore, since $L_{F}=\left[\rho\left(F_{l}\right), \rho\left(F_{u}\right)\right]$ and $\rho\left(F_{\mu}\right)$ depends continuously on $\mu$ (see, for instance, [1, Lemma 3.7.12]) there are $\mu_{a}<\mu_{b}$ such that $\rho\left(F_{\mu_{a}}\right)=a$ and $\rho\left(F_{\mu_{b}}\right)=b$. By Lemma 3.7.16 and Proposition 3.7.17 of [1] there are twist cycles (mod. 1) $P$ and $Q$ of $F$ such that $\left.F\right|_{P}=\left.F_{\mu_{a}}\right|_{P}$ and $\left.F\right|_{Q}=\left.F_{\mu_{b}}\right|_{Q}$. We have $\rho(P)=a$ and $\rho(Q)=b$.

There are points $Y \in Q$ and $Z \in P$ such that $Y<Z$ and $(Y, Z) \cap(P \cup Q)=$ $\emptyset$. If $i \in\{1,2\}$ then $b>p_{i} / q_{i}$, so the graph of $\left(F_{\mu_{b}}\right)^{q_{i}}-p_{i}$ lies above the diagonal. Therefore if $T \in Q$ then $F^{q_{i}}(T)>T+p_{i}$. Similarly, if $T \in P$ then $F^{q_{i}}(T)<T+p_{i}$. In particular, if we set $V=F^{q_{2}}(Y)-p_{2}$ then $V>Y$. Since there are no points of $P \cup Q$ between $Y$ and $Z$, and $P \cap Q=\emptyset$, we get $V>Z$. Moreover, $F^{q_{2}}(Y)=V+p_{2}>Z+p_{2}, F^{q_{2}}(Z)<Z+p_{2}, F^{q_{1}}(Z)<Y+p_{1}$ and $F^{q_{1}}(V)>V+p_{1}>Z+p_{1}$. Therefore $Y<Z<V$, the points $Y, Z, V$ belong to $P \cup Q,\left[Z+p_{2}, V+p_{2}\right] \subset F^{q_{2}}([Y, Z])$ and $\left[Y+p_{1}, Z+p_{1}\right] \subset F^{q_{1}}([Z, V])$. Hence, for each map $G \in \mathcal{L}$ such that $\left.G\right|_{P \cup Q}=\left.F\right|_{P \cup Q}$ there exists a loop of $(P \cup Q)$-basic intervals of length $q_{1}+q_{2}$, beginning at $[Y, Z]$ and ending at $\left[Y+p_{1}+p_{2}, Z+p_{1}+p_{2}\right]$, and such that the $q_{2}$-th interval of this loop (if $[Y, Z]$ is 0 -th) is contained in $\left[Z+p_{2}, V+p_{2}\right]$. This loop gives us a cycle (mod. 1) $R$ of $G$ with the properties listed in (i). Notice that the inequality $G^{q_{2}}(X)>X+p_{2}$ is strict since $Z \notin R$, and $Z \notin R$ holds because $Z \in P$ and the $G$-rotation numbers of $P$ and $R$ are different.

Now we prove (ii). Set $\widetilde{F}=\max \left(\min \left(F_{\mu_{b}}, F\right), F_{\mu_{a}}\right)$. Clearly, $\widetilde{F} \in \mathcal{L}$. Since $\left.F_{\mu_{b}}\right|_{Q}=\left.F\right|_{Q}$ and $F_{\mu_{a}} \leq F_{\mu_{b}}$, we get $\left.\widetilde{F}\right|_{Q}=\left.F\right|_{Q}$. One can check by inspection that for every $x, y, z \in \mathbb{R}$, if $x \leq z$ then $\max (\min (z, y), x)=$ $\min (\max (x, y), z)$. Therefore, for $\widetilde{F}$ we also have the formula $\widetilde{F}=$ 
$\min \left(\max \left(F_{\mu_{a}}, F\right), F_{\mu_{b}}\right)$. Then we get $\left.\widetilde{F}\right|_{P}=\left.F\right|_{P}$ in a similar way as for $Q$. Thus $\left.\widetilde{F}\right|_{P \cup Q}=\left.F\right|_{P \cup Q}$.

From Theorem 3.7.20 of [1] we have $F_{\mu_{a}} \leq \widetilde{F} \leq F_{\mu_{b}}$, so the rotation interval of $\widetilde{F}$ is contained in $[a, b]$. However, $P$ and $Q$ are cycles (mod. 1) of $\widetilde{F}$, and they have rotation numbers $a$ and $b$, respectively. Therefore $L_{\widetilde{F}}=$ $[a, b]$.

Notice that the above lemma does not say what the period of $R$ is. The only thing we know is that this period divides $q_{1}+q_{2}$. However, the exact knowledge of the period of $R$ is not necessary for proving the next two lemmas.

Lemma 5.3. Under the assumptions of Lemma 5.2, assume additionally that $p_{1}=r_{1}+m r, p_{2}=r_{2}+k r, q_{1}=s_{1}+m s, q_{2}=s_{2}+k s$, where $k, m$ are non-negative integers with $m+k=n-1$, and $n, r, s, r_{1}, r_{2}, s_{1}, s_{2}$ are as in the definition of $\alpha_{r / s}$ and $\beta_{r / s}$ (in particular, $s>1$ ). Then the cycle (mod. 1 ) $R$ from Lemma 5.2 is badly ordered.

Proof. Assume that $R$ is not badly ordered. Then $R$ has a block structure over a twist cycle $S$ of a map $\varphi \in \mathcal{L}$. In view of Lemma 4.2 we see that $\rho_{\varphi}(S)=\rho_{F}(R)=\left(p_{1}+p_{2}\right) /\left(q_{1}+q_{2}\right)=(n r) /(n s)=r / s$. Let $R_{i}$ denote the blocks of $R$ from the definition of a block structure. Without loss of generality we may assume that for each $Y \in R_{i}$ and $Z \in R_{j}$ we have $Y<Z$ if and only if $i<j$ and that $X \in R_{0}$ (where $X$ is the point from Lemma 5.2). Then, by Lemma 5.1, $F\left(R_{i}\right)=R_{i+r}$ for each $i \in \mathbb{Z}$. Hence, $F^{q_{2}}(X) \in R_{q_{2} r}$. On the other hand we note that, since $F^{q_{2}}(X)-p_{2}>X$, we have $F^{q_{2}}(X) \in$ $R_{i}+p_{2}$ with $i \geq 0$. Consequently, $R_{q_{2} r}=R_{i}+p_{2}$. Since $S$ has period $s$ and is twist, we have $R_{i}+p_{2}=R_{i+p_{2} s}$. Thus, $q_{2} r=p_{2} s+i$ which implies that $r / s \geq p_{2} / q_{2}$. But, since $p_{2} / q_{2}=\left(r_{2}+k r\right) /\left(s_{2}+k s\right)$ and $r / s<r_{2} / s_{2}$ we get that $r / s<p_{2} / q_{2}$, a contradiction. Therefore $R$ is badly ordered.

Lemma 5.4. Under the assumptions of Lemma 5.2, assume additionally that $p_{1}=q_{1} r-1$ and $p_{2}=q_{2} r+1$ for some $r \in \mathbb{Z}$. Then the cycle (mod. 1$)$ $R$ from Lemma 5.2 is badly ordered.

Proof. Suppose that $R$ is not badly ordered. Since $p_{1}+p_{2}=\left(q_{1}+q_{2}\right) r$, the rotation number of $R$ is an integer, so by Lemmas 4.2 and 5.1, $R$ has a block structure over a cycle (mod. 1) of period 1. The diameter of each block has to be smaller than 1 . The block to which $X$ belongs (where $X$ is the point from Lemma 5.2) consists of all the points of the form $F^{n}(X)-n r, n \in \mathbb{Z}$. However, $F^{q_{2}}(X)-q_{2} r>X+p_{2}-q_{2} r=X+1$, so the diameter of this block is larger than 1 , a contradiction. Therefore $R$ is badly ordered. 
The last ingredient of the proof of Theorem $\mathrm{C}$ is the comparison of the intervals appearing in the statement of Theorem A for various values of $n$.

Lemma 5.5. If either $\left[\alpha_{r / s}\left(m^{\prime}\right), \beta_{r / s}\left(k^{\prime}\right)\right] \subset \quad\left[\alpha_{r / s}(m), \beta_{r / s}(k)\right]$ or $\left[r-\frac{1}{m^{\prime}}, r+\frac{1}{k^{\prime}}\right] \subset\left[r-\frac{1}{m}, r+\frac{1}{k}\right]$, then $m^{\prime}+k^{\prime} \geq m+k$.

Proof. As it was observed at the beginning of Section 3, the function $\alpha_{r / s}$ is increasing and $\beta_{r / s}$ is decreasing. Therefore if $\left[\alpha_{r / s}\left(m^{\prime}\right), \beta_{r / s}\left(k^{\prime}\right)\right] \subset\left[\alpha_{r / s}(m)\right.$, $\left.\beta_{r / s}(k)\right]$ then $m^{\prime} \geq m$ and $k^{\prime} \geq k$. Hence, $m^{\prime}+k^{\prime} \geq m+k$. The proof when $\left[r-\frac{1}{m^{\prime}}, r+\frac{1}{k^{\prime}}\right] \subset\left[r-\frac{1}{m}, r+\frac{1}{k}\right]$ is similar.

Now we are ready to prove Theorem C.

Proof of Theorem C. If $s>1$ then we set $p_{1}=r_{1}+m r, p_{2}=r_{2}+k r$, $q_{1}=s_{1}+m s$ and $q_{2}=s_{2}+k s$, where $r_{1}, r_{2}, s_{1}, s_{2}$ are as in the definition of $\alpha_{r / s}$ and $\beta_{r / s}$. If $s=1$ then we set $q_{1}=m, q_{2}=k, p_{1}=q_{1} r-1$ and $p_{2}=q_{2} r+1$. Notice that then $\left[p_{1} / q_{1}, p_{2} / q_{2}\right]$ is the interval from the statement of the theorem, contained in the interior of $L_{F}$. Then we choose $a, b \in \mathbb{Q}$ such that $a<p_{1} / q_{1}<p_{2} / q_{2}<b$ and $[a, b] \subset L_{F}$ and we apply Lemma 5.2. By Lemmas 5.3 (if $s>1$ ) and 5.4 (if $s=1$ ), the cycle (mod. 1) $R$ that we obtain, is badly ordered. It remains to prove that its period is $q_{1}+q_{2}=n s$. This is not necessarily true under the assumptions we made up to now, but we will show that with more careful choice of $a$ and $b$ it will hold.

The period of $R$ divides $n s$, and since the rotation number of $R$ is $r / s$ with $r$ and $s$ coprime, it is a multiple of $s$. Therefore it is $l s$ for some $l \leq n$. Look at the map $\widetilde{F}$ from Lemma 5.2 . It has a cycle with the same properties as $R$. By Theorem A, the rotation interval of $\widetilde{F}$ (which is $[a, b]$ by Lemma 5.2) contains an interval of the form $\left[\alpha_{r / s}\left(m^{\prime}\right), \beta_{r / s}\left(k^{\prime}\right)\right]$ for some non-negative integers $m^{\prime}, k^{\prime}$ with $m^{\prime}+k^{\prime}=l-1$ if $s>1$ and of the form $\left[r-\frac{1}{m^{\prime}}, r+\frac{1}{k^{\prime}}\right]$ for some positive integers $m^{\prime}, k^{\prime}$ with $m^{\prime}+k^{\prime}=l$ if $s=1$. There is only finitely many choices for $l, m^{\prime}, k^{\prime}$, so by choosing $a$ sufficiently close to $p_{1} / q_{1}$ and $b$ sufficiently close to $p_{2} / q_{2}$ we can eliminate those choices for which the assumptions of Lemma 5.5 are not satisfied. Hence by Lemma $5.5, l \geq n$. This means that $l=n$, so the period of $R$ is $n s$.

\section{References}

[1] L. Alsedà, J. Llibre and M. Misiurewicz, Combinatorial dynamics and entropy in dimension one, Advanced Series on Nonlinear Dynamics, 5, World Scientific, Singapore, 1993.

[2] L. Alsedà, J. Llibre, M. Misiurewicz and C. Simó, Twist periodic orbits and topological entropy for continuous maps of the circle of degree one which have a fixed point, Ergod. Th. \& Dynam. Sys., 5 (1985), 501-517. 
[3] P.L. Boyland, Rotation sets and monotone periodic orbits for annulus homeomorphisms, Comment. Math. Helvetici, 67 (1992), 203-213.

[4] P.L. Boyland and G.R. Hall, Invariant circles and the order structure of periodic orbits in monotone twist maps, Topology, 26 (1987), 21-35.

[5] A. Chenciner, J.-M. Gambaudo and C. Tresser, Une remarque sur la structure des endomorphismes de degré 1 du cercle, C. R. Acad. Sci. Paris, Sér. I Math., 299 (1984), 145-148.

[6] R. Ito, Rotation sets are closed, Math. Proc. Camb. Phil. Soc., 89 (1981), 107-111.

[7] R.S. MacKay and C. Tresser, Badly ordered orbits of circle maps, Math. Proc. Camb. Phil. Soc., 96 (1984), 447-451.

[8] S. Newhouse, J. Palis and F. Takens, Bifurcations and stability of families of diffeomorphisms, Inst. Hautes Études Sci. Publ. Math., 57 (1983), 5-71.

Received October 16, 1996.

Universitat Autònoma de Barcelona

08193 Cerdanyola del Vallès, Barcelona

SPAIN

E-mail address: alseda@mat.uab.es

IUPUI

INDIANAPOLIS, IN 46202-3216

E-mail address: mmisiure@math.iupui.edu

and

Universitat Autònoma de BARCELONA

08193 Cerdanyola del Vallès, Barcelona

SPAIN

E-mail address: jllibre@mat.uab.es 